La Revue

des Droits

de l'Homme

\section{La Revue des droits de l'homme}

Revue du Centre de recherches et d'études sur les droits fondamentaux

$10 \mid 2016$

Revue des droits de l'homme - $\mathrm{N}^{\circ} 10$

\title{
« ...les droits et libertés que la constitution garantit » : quiproquo sur la QPC?
}

\section{Stéphanie Hennette Vauchez}

\section{OpenEdition \\ Journals}

Édition électronique

URL : http://journals.openedition.org/revdh/2481

DOI : $10.4000 /$ revdh.2481

ISSN : 2264-119X

\section{Éditeur}

Centre de recherches et d'études sur les droits fondamentaux

\section{Référence électronique}

Stéphanie Hennette Vauchez, « « ...les droits et libertés que la constitution garantit » : quiproquo sur la QPC ? ", La Revue des droits de l'homme [En ligne], 10 | 2016, mis en ligne le 11 juillet 2016, consulté le 24 juillet 2020. URL : http://journals.openedition.org/revdh/2481; DOI : https://doi.org/10.4000/revdh. 2481

Ce document a été généré automatiquement le 24 juillet 2020.

Tous droits réservés 


\title{
«...les droits et libertés que la constitution garantit » : quiproquo sur la QPC?
}

\author{
Stéphanie Hennette Vauchez
}

1 Six ans déjà̀ ${ }^{1}$ La QPC a atteint, c'est probable, quelque chose comme un rythme de croisière. Si les premières années suivant l'entrée en vigueur de la nouvelle procédure ont pu être marquées, aux dires de certains acteurs et observateurs, par des usages spécifiques de la QPC liés à l'effet d'aubaine et à l'appel d'air crées par la nouveauté, on peut gager qu'il en va désormais différemment que la procédure est routinisée. On cherche dès lors à interroger ici la QPC ordinaire, celle qui est entrée dans les mœurs juridiques. Et on souhaite le faire dans un but bien précis: confronter une année normale, banale, de QPC aux promesses et programmes auxquels elle a, depuis sa création, été associée -lesquels sont ambitieux. La QPC nous est ainsi présentée comme «la principale avancée des droits et libertés dans notre pays depuis l'abolition de la peine de mort $»^{2}$; l'article $61 \$ 1$ lui assigne pour mission, rien de moins, d'assurer la protection des « droits et libertés que la Constitution garantit ».

2 Les analyses présentées reposent sur l'étude systématique d'une année de contentieux QPC -la dernière année écoulée (avril 2015-avril 2016). Travailler sur une année de contentieux QPC permet de procéder à un travail à la fois quantitatif et qualitatif. Quantitatif, parce que les 66 décisions QPC rendues par le Conseil constitutionnel permettent d'entamer un travail de mise en ordre statistique, et d'identifier des tendances : quelles sont les dispositions mises en cause, par qui, sur le fondement de quels moyens? Qualitatif, car la taille encore mesurée du corpus d'analyse permet de conjurer les effets d'invisibilisation liés au maniement des grandes masses. Ainsi par exemple, le récent bilan de 5 ans de QPC réalisé par le Conseil constitutionnel indique que les décisions de censure concernent en premier lieu le droit pénal et la procédure pénale, et en second lieu le droit fiscal et la procédure fiscale ${ }^{3}$. Mais pour réellement comprendre la mesure dans laquelle la QPC contribue à la protection «des droits et libertés que la constitution garantit ", il importe de s'affranchir de ces classifications, 
car seule une approche qualitative reposant sur l'analyse substantielle des décisions permet de mesurer l'extraordinaire plasticité de cette catégorie centrale de la QPC. On entend démontrer que les «droits et libertés que la Constitution garantit » renvoient, en fait, à un ensemble non seulement hétérogène mais aussi contre-intuitif. On y trouve bien sûr des questions substantielles essentielles à la protection des personnes. Mais on y trouve aussi des questions très éloignées; on constate même que la QPC peut être mise au service de finalités ou objectifs que l'on pourrait considérer comme opposés à une compréhension intuitive de la catégorie «droits et libertés que la Constitution garantit $»^{4}$.

3 Au principe de la démarche proposée réside un doute. Le doute qu'en matière de QPC, un piège des mots joue à plein -un piège qui voudrait que le vocable même dans lequel la QPC s'énonce (une possibilité ouverte à toute personne de contester des dispositions législatives méconnaissant "les droits et libertés que la Constitution garantit») ne serait que trompeusement universaliste -abstrait, neutre. Le doute que les «droits et libertés que la Constitution garantit » ne sont pas forcément ceux auxquels il est implicitement renvoyé lorsqu'acteurs et observateurs de la QPC invoquent, pêle-mêle, une nouvelle étape de la diffusion d'une culture des libertés à l'ensemble de l'ordre juridique, la consécration d'une "sentinelle démocratique" de l'État de droit ${ }^{5}$, "l'entrée de l'individu dans l'espace constitutionnel où se discutent, s'acquièrent, se forment ses droits et libertés ${ }^{6}$. D'où l'hypothèse que l'analyse des usages sociaux de la QPC (qui soulève effectivement des QPC? dans quels buts et en invoquant quels moyens ?) est essentielle à une compréhension véritable de ce que la QPC protège vraiment -de ce que recouvre réellement cette catégorie des «droits et libertés que la Constitution garantit ${ }^{7}$.

Qui soulève des QPC ? En matière de QPC, les requérants jouent un rôle déterminant dans la délimitation du contentieux ${ }^{8}$; dans ces conditions, on peut formuler l'hypothèse selon laquelle une plus grande proportion de certaines catégories de requérants (comme par exemple les entreprises), qui feront plus classiquement et plus logiquement valoir certains moyens que d'autres (comme par exemple les libertés économiques), inscrit la procédure même sur une pente. Car en effet, si un texte -fût-il énoncé constitutionnel- ne veut rien dire et que sa portée réelle dépendra toujours, in fine, de l'interprétation qui en sera faite, cette dernière est dépendante, en amont, des usages sociaux de l'énoncé : sera-t-il invoqué, contesté, mobilisé, par qui, et dans quel but? Ainsi, selon qu'il est tendanciellement mobilisé par des détenus contestant des conditions de détention contraires à la dignité humaine ou par des entreprises cherchant à mettre en cause la loi fiscale ${ }^{9}$, un même énoncé recevra une tonalité et des interprétations fort différentes. En cela, un regard sur les usages sociaux de la QPC permet de souligner à nouveau les enjeux associés à la question de l'accès au droit. S'il peut, dans certaines configurations historiques spécifiques, être un outil et un vecteur des luttes et des revendications sociales, le droit est en effet un instrument ambivalent : complexe, menaçant, perçu comme ou effectivement coûteux à mobiliser, le droit peut aussi aisément être lu comme un instrument de domination -un instrument des élites, des puissants, au service des puissants. La QPC est-elle une arme supplémentaire des citoyens pour obtenir la protection de leurs droits constitutionnels? A-t-elle fait du citoyen un «acteur constitutionnel $»^{10}$ ? L'examen du corpus ici constitué ne permet pas de l'affirmer. 
Quels sont les «droits et libertés que la Constitution garantit " dont on cherche à obtenir la protection par le biais de la QPC? On observe ici que les entreprises sollicitent davantage la QPC pour contester la réglementation applicable à tel ou tel segment de marché en invoquant la liberté d'entreprendre que des victimes de harcèlement sexuel ne le font en invoquant l'égalité entre les sexes et le droit à la protection de leur intégrité physique. Il ne s'agit pas, ce faisant, de dénoncer une prétendue instrumentalisation des droits et libertés, ni même de remettre en cause l'affirmation selon laquelle les personnes morales sont titulaires de droits fondamentaux. Sans rouvrir ce débat ${ }^{11}$, on souhaite insister sur les effets des usages sociaux de la QPC sur la coloration du contentieux -sa tonalité, son centre de gravité. On peut d'ailleurs faire un parallèle avec d'autres terrains juridiques : il y a quelques années, des travaux menés sur la notion de droits fondamentaux à l'échelle européenne et sur l'apport comparé des deux juridictions européennes à leur protection avaient déjà révélé une forme de path dependency luxembourgeoise. Ainsi, l'inscription de la protection des droits fondamentaux à l'agenda de l'Union n'avait pas réussi à altérer l'inclinaison économique du droit de l'Union européenne, due non seulement au contenu matériel des normes européennes mais aussi, bien plus largement, à l'identité et aux intérêts des acteurs ancrés dans le droit de l'Union. Laurent Scheeck établissait qu'à Luxembourg et Strasbourg, un même texte (la Convention Européenne des droits de l'Homme) était mobilisé bien différemment, qu'en conséquence, l'objet «droits fondamentaux » différait sensiblement dans son contenu ici et là, largement mobilisé qu'il était, à Luxembourg, au service de la libre concurrence ${ }^{12}$. S'il ne s'agit pas de présenter de manière simpliste les intérêts économiques et les droits de l'Homme comme deux sphères étanches et insusceptibles de coïncider ${ }^{13}$, il n'en reste pas moins vrai qu'ils sont de nature à entrer en conflit ${ }^{14}$.

Pour tester ces hypothèses de travail, on ne s'intéresse pas à titre principal au Conseil constitutionnel (ni l'institution ${ }^{15}$, ni la jurisprudence). On ne cherche pas non plus à mesurer ou à comprendre ce que la QPC produit en termes de droit constitutionnel. On souhaite plutôt saisir la portée des QPC telles qu'elles sont soulevées par les requérants. On espère ainsi contribuer à cerner ce que la $\mathrm{QPC}$ en est venue à représenter, après six années d'existence, dans le monde du droit : qui soulève des QPC ? Pour contester quel type d'énoncés? En soulevant quels moyens? Voilà, sommairement ramassées, les interrogations qui ont guidé le présent travail, entendu comme proposant des pistes de recherche destinées, le cas échéant, à être approfondies, étendues et systématisées. Les premières conclusions tirées de l'étude tendent à souligner la très forte représentation, parmi les auteurs de QPC sur la période analysée, d'entreprises et fédérations d'entreprise, soulevant abondamment le principe d'égalité, des garanties procédurales ainsi que les libertés économiques (liberté d'entreprendre, droit de propriété, liberté contractuelle), pour obtenir l'abrogation des dispositions très souvent fiscales et/ou de droit des affaires, voire celle de dispositions et dispositifs qui seraient volontiers assimilés à des droits de l'Homme par nombre d'acteurs sociaux. Ainsi, les «droits et libertés que la Constitution garantit ", catégorie juridique nouvelle née à la faveur de la $\mathrm{QPC}$, ne sont pas forcément ceux que l'on croit -et l'on suggère que ceci n'est pas sans rapport avec le fait que l'outil QPC sert plus souvent à la Sté Uber qu'à la Ligue des Droits de l'Homme. On présente ces résultats en examinant sous ses différentes facettes cette catégorie centrale à la QPC que sont les «droits et libertés que la Constitution garantit ». On dressera ainsi d'abord le portrait du justiciable soulevant une QPC (I.), pour chercher ensuite à comprendre l'objectif qu'il poursuit, en s'attachant à la nature 
des dispositions attaquées (II.). On s'attarde ensuite sur le rôle des filtres (juge a quo, Conseil d'Etat, Cour de cassation) qui opèrent entre le justiciable et le Conseil constitutionnel, dans la mesure où ils participent eux aussi largement au façonnage de la catégorie "droits et libertés que la Constitution garantit » (III.). On s'intéresse enfin aux moyens invoqués dans le contentieux QPC : quels sont ces droits et libertés dont les justiciables estiment qu'ils sont méconnus (IV.) ?

\section{Portrait du justiciable de la QPC : qui demande la protection des « droits et libertés que la Constitution garantit »?}

7 Des entreprises et des hommes : voilà qui, dans le corpus étudié, soulève des QPC. S'il s'agit à peu près à égalité de personnes morales et de personnes physiques, les entreprises dominent clairement la première catégorie et les hommes, la seconde ${ }^{16}$. Ce n'est que significativement loin derrière qu'apparaissent à l'origine de QPC des ONG, des syndicats, ou des femmes. Il y a donc bien un profil dominant du justiciable mobilisant l'outil QPC. La lecture peut encore être affinée, car dans la procédure de QPC, il existe d'autres statuts que celui de requérant : des tiers au litige ayant donné lieu à la QPC peuvent ainsi intervenir en défense ou, plus simplement encore, comme simples intervenants ${ }^{17}$. À ce titre, on a recensé sur le corpus étudié : 6 interventions d'entreprises ou fédérations d'entreprises, 5 interventions d'ONG ${ }^{18}$ (LICRA et MRAP, LDH, GISTI, OIP, Fondation France Libertés) et 3 interventions de syndicats (CGPME, FO et CGT FO). Ces premières données sont déjà intéressantes. Elles permettent notamment d'établir les points suivants.

Pour ce qui est personnes physiques qui sont à l'origine des décisions QPC rendues par le Conseil constitutionnel sur la période étudiée, s'il est plus difficile de proposer une lecture fine en l'absence de tout autre élément d'information que le sexe des requérants, on ne saurait néanmoins passer sous silence ce que révèle déjà ce simple critère : sur 34 QPC initiées par des personnes physiques, 26 l'ont été par des hommes, contre seulement 4 par des femmes -dont l'une est, en outre, une femme politique jouant, par le biais de la QPC, la continuation sur le terrain du droit de la bataille politique $^{19}$. Parmi ces QPC soulevées par des requérantes femmes, deux affaires portaient sur des droits et protections sociales ${ }^{20}$, ce qui n'est pas inintéressant au regard du fait que l'analyse féministe du droit n'a de cesse de souligner la marginalité des questions sociales et, plus largement, de tout ce qui ressortit de la sphère privée, dans le façonnement juridique de la réalité sociale ${ }^{21}$.

Pour ce qui est personnes morales, il apparait assez clairement que si le prétoire du Conseil constitutionnel s'est, mécaniquement, ouvert suite à la création de la QPC comme nouvelle voie de recours, les entreprises et fédérations d'entreprise occupent bien en effet une place de choix dans le contentieux QPC de la période étudiée ${ }^{22}: 24$ recours formés, c'est plus de $36 \%$ du total. A l'inverse, on peut souligner la faible part occupée par les ONG et associations -le pôle "droits de l'Homme» du contentieux considéré : 4 recours, c'est ici $6 \%$ du total. Pire encore pour les syndicats : 1 seul recours pour la période considérée, soit un pourcentage infime. Premier enseignement de cette dissection d'une année de QPC, dès lors : la défense d'intérêts collectifs par des personnes morales n'est que faiblement ou marginalement celle de structures et entités 
qui font de cette défense leur raison sociale. Au contraire, les syndicats et ONG de défense des droits de l'Homme étant ici très faiblement présents, ce sont les entreprises qui, dans des proportions importantes, s'emparent de l'outil « droits et libertés que la Constitution garantit " pour défendre leurs intérêts collectifs -à connotation souvent économique. Comme l'a déjà écrit Xavier Dupré de Boulois, à la faveur de la QPC, les opérateurs économiques voient «la contrée des droits fondamentaux" comme "un vaste supermarché où chacun pioche des ressources argumentatives pour soutenir ses intérêts économiques $»^{23}$.

10 Comme en écho à cette physionomie singulière du groupe des requérants sur l'année étudiée, on est frappé par la table des matières du numéro 137 de la revue Pouvoirs qui, consacrant en 2011 une livraison à la QPC, envisageait de manière séparée "L'appropriation de la QPC par ses acteurs " ${ }^{24}$ et « La QPC et les citoyens »" Au-delà de cette distinction qui véhicule, implicitement, l'idée que les uns ne sont pas les autres que les citoyens ne comptent pas directement parmi les acteurs de la QPC-, Emmanuel Piwnica, avocat au Conseil d'Etat et à la Cour de Cassation et auteur de l'article sur les acteurs de la QPC, entreprenait de lister les acteurs de la QPC. On trouve dans l'énumération proposée « ceux qui la jugent, les membres du Conseil constitutionnel, les membres du Conseil d'Etat et les magistrats de la Cour de cassation, l'ensemble des juges administratifs ou judiciaires; ceux qui la posent : les avocats au Conseil d'Etat et à la Cour de cassation et les avocats près les cours et tribunaux; sans oublier ceux qui la commentent: les professeurs de droit $\rrbracket^{26}$-mais pas les citoyens. Olivier Duhamel, qui s'intéressait au rapport entre la QPC et les citoyens, concédait quant à lui qu'elle ne confère pas tant "un pouvoir au citoyen» qu'un «droit au justiciable $»^{27}$, et se concentrait sur des aspects symboliques (construction d'une salle d'audience ouverte au public, déploiement des outils numériques de communication...) pour convaincre de l'intérêt de la QPC pour le citoyen...

\section{Profil des objectifs poursuivis par la voie de la QPC : de quelles dispositions l'abrogation est-elle demandée au motif de leur contrariété aux « droits et libertés que la Constitution garantit »?}

11 Sur l'année étudiée, les requérants sont donc en large part des entreprises ou autres personnes morales intéressées par la vie économique. Par-delà ces éléments de profiltype des requérants, il importe, pour saisir les usages sociaux de la QPC, de comprendre quels sont les objectifs poursuivis par les auteurs de ce recours : quelles sont les normes dont ils questionnent la constitutionnalité et en quoi sont-elles réputées méconnaître « les droits et libertés que la Constitution garantit »? En d'autres termes, que révèlent les usages sociaux de la QPC de la réalisation (ou non) du principal objectif de la révision constitutionnelle de 2008 qui était d'accroître et intensifier la protection constitutionnelle des droits et libertés fondamentales?

12 En l'occurrence, le travail de dissection d'un an de QPC permet d'établir, bien à contrecourant de l'antienne qui, depuis l'instauration de la QPC, la décrit et la définit comme un vecteur de diffusion des droits et libertés dans l'ensemble du monde juridique, que les règles dont les justiciables cherchent à voir l'inconstitutionnalité déclarée via la nouvelle procédure sont, pour l'essentiel, bien éloignées de ce domaine des « droits et 
libertés $»^{28}$. En d'autres termes, c'est bien plutôt pour faire progresser un agenda fiscal ou de droit des affaires que les requérants utilisent la QPC; c'est parfois aussi pour contester des dispositions contraignantes contenues dans le Code du travail. Mais le domaine des libertés des personnes (liberté d'expression, protection de la liberté individuelle, articulation liberté / sécurité...) occupe ici la portion congrue. On propose, pour plus de clarté, la typologie ci-dessous :

\begin{tabular}{|l|l|}
\hline Domaine & Proportion (sur 66 décisions dépouillées) \\
\hline Droit fiscal & 16 décisions \\
\hline Droit des affaires et de l'économie & 12 décisions \\
\hline Sous-total 1 & 28 décisions (42,42\%) \\
\hline Droit du travail & 8 décisions \\
\hline Sécurité et Liberté & 8 décisions \\
\hline Procédure judiciaire & 6 décisions \\
\hline Sous-total 2 & 22 décisions (33,3\%) \\
\hline Varia & 15 décisions \\
\hline
\end{tabular}

13 Les catégories que l'on a retenues pour classer une année de contentieux QPC sont fondées sur l'analyse qualitative, substantielle, du corpus. Ainsi, dépassant une logique purement formelle qui consisterait à présenter comme "pénale » toute QPC visant à mettre en cause une disposition du Code pénal, on a retenu comme déterminante non pas la source de la disposition attaquée (le Code pénal, le Code du commerce...) mais sa substance. Il en résulte une catégorie «Droit fiscal» qui regroupe l'ensemble des dispositions où étaient contestés des mécanismes d'imposition et de contribution ${ }^{29}$. Selon la même logique, on isole ici une catégorie "Droits des affaires et de l'économie " qui rassemble des recours formés contre la réglementation des marchés ${ }^{30}:$ règles pesant sur les acteurs (ex. : recours contre la réglementation des points de vente de presse $^{31}$ ) ou les produits (ex.: interdiction de la fabrication et de l'exportation de produits contenant du bisphénol $\mathrm{A}^{32}$ ), mécanismes de sanction du non-respect de ces règles (ex. : cumul de poursuites pénales et boursières (AMF) ${ }^{33}$ ). On atteint un total de 28 décisions, soit plus de $42 \%$ du corpus étudié, suscitées par des procédures de QPC intentées dans le but de remettre en cause la constitutionnalité de dispositions législatives à nature fiscale ou de réglementation et régulation de l'activité économique et des marchés. Voilà qui donne une teinte singulière au contentieux de la QPC, et invite à repenser la contribution de cette procédure à l'intensification de la protection constitutionnelle des droits et libertés en France.

Par-delà ces éléments chiffrés, on peut illustrer ce qui précède en centrant la focale sur quelques cas spécifiques. On fait le choix d'extraire du corpus étudiés deux exemples. Un premier exemple est constitué d'une QPC choisie pour la manière dont elle illustre combien cette voie de recours peut aisément être mobilisée à rebours de dispositions relatives à la protection des droits ${ }^{34}$. Un second exemple est constitué de l'ensemble des 
QPC soulevées, dans le corpus étudié, à l'encontre de dispositions du Code du travail ; il permet d'établir l'ambivalence de l'outil, certainement potentiellement mis au service de la protection des «droits et libertés » des salariés, mais potentiellement aussi, au service du détricotage des règles protectrices.

La décision 2015-470 QPC fait suite à la QPC soulevée par la Société Saur SAS contre la dernière phrase du troisième alinéa de l'article L. 115-3 du code de l'action sociale et des familles. Cette disposition interdit aux fournisseurs d'électricité, de chaleur et de gaz de procéder à l'interruption de leurs services dans les résidences principales de leurs clients entre le $1^{\text {er }}$ novembre et le $1^{\text {er }}$ mars de chaque année. Depuis la loi $\mathrm{n}^{\circ}$ 2007-290 du 5 mars 2007 instituant le droit au logement opposable et portant diverses mesures en faveur de la cohésion sociale, cette disposition du CASF prévoit en outre que cette interdiction s'applique aussi aux fournisseurs d'eau et ce, tout au long de l'année. «Acteur historique de la gestion déléguée de services à l'environnement, le Groupe 䣑Saur SAS熙 accompagne les collectivités locales et les industriels dans leurs projets liés à l'eau, la propreté, l'ingénierie, les travaux, les loisirs et le multiservices » ${ }^{35}$. Pour ce faire, il a entrepris de contester par le biais de la QPC l'interdiction qui lui est faite, comme aux autres fournisseurs d'eau, de procéder à l'interruption du service pour défaut de paiement des clients. À titre principal, la société requérante faisait ainsi valoir devant le Conseil constitutionnel que l'article L. 115-3 du CASF porte une " atteinte excessive, d'une part, à la liberté contractuelle et à la liberté d'entreprendre et, d'autre part, aux principes d'égalité devant la loi et les charges publiques ». Le requérant n'a pas convaincu le Conseil. Celui-ci a en effet considéré en premier lieu que les dispositions attaquées entendaient empêcher l'interruption du service de l'eau afin d'assurer la mise en œuvre de l'objectif à valeur constitutionnelle de droit de toute personne à un logement décent (lequel justifie, par ailleurs, que des règles différentes s'appliquent à l'eau d'une part et au gaz ou à l'électricité d'autre part). Il a précisé en second lieu que la distribution et la tarification de l'eau opèrent toutes deux sur un marché réglementé, encadré par la loi, caractérisé notamment par le fait que l'usager n'a pas le choix de son distributeur, de sorte que l'atteinte à la liberté contractuelle et à la liberté d'entreprendre ne saurait être regardée comme disproportionnée. Cette décision est néanmoins intéressante pour ce qu'elle fait entrevoir des usages de la QPC par différents acteurs économiques.

168 des 66 décisions QPC rendues par le Conseil constitutionnel sur la période étudiée trouvent leur origine dans la contestation de dispositions du Code du travail; elles illustrent bien l'ambivalence de l'outil QPC au regard de l'objectif de la protection des droits de l'Homme. Parmi ces 8 décisions, deux portent sur des enjeux de représentativité des organisations professionnelles ${ }^{36}$. S'il s'agit indubitablement d'un enjeu important au regard de la notion même de démocratie sociale, ce ne sont pas des affaires mettant directement en cause les droits des personnes ${ }^{37}$. Idem pour une autre de ces 8 QPC, qui porte sur les règles relatives au droit à perception de la taxe d'apprentissage ${ }^{38}$. En d'autres termes, le nombre de ces QPC 'Code du travail' portant directement sur les droits des personnes est, en fait, réduit à 5 dans l'échantillon concerné. Parmi ces 5 décisions, deux mettaient en cause les droits des salarié(e)s : la première restaure le droit à indemnité compensatrice de congés payés, même lorsque la rupture du contrat de travail est causée par la faute lourde du salariée ${ }^{39}$, tandis que la seconde donne l'occasion au Conseil de juger qu'une loi du 19 juillet 2010 relative à la santé au travail adoptée par l'assemblée de Polynésie française poursuivait un objectif d'intérêt général (permettre une indemnisation automatique, rapide et sûre) en 
limitant la réparation de l'accident du travail dû à la faute inexcusable de l'employeur au seul versement d'une indemnité forfaitaire ${ }^{40}$. Requérants-salariés victorieux ici, défaits là : la QPC ne change peut être pas le visage du droit constitutionnel du travail ; une spécialiste juge d'ailleurs que la question des droits fondamentaux y demeure "secondaire " $^{41}$. À ceci près que la QPC offre tout de même aux employeurs une voie nouvelle pour contester nombre de règles protectrices des travailleurs. Qu'on en juge : les trois dernières affaires des 8 QPC mettant en cause des dispositions du Code du travail dans le corpus étudié trouvaient ainsi leur origine dans des procédures intentées par diverses entreprises (la fédération des promoteurs immobiliers ${ }^{42}$, la Société Gecop ${ }^{43}$, et un groupement d'employeurs ${ }^{44}$ ) dans le but d'obtenir des décisions d'inconstitutionnalité de dispositions relatives à la responsabilité des employeurs en cas de conditions d'hébergement contraires à la dignité, aux modalités de calcul de l'obligation d'emploi de travailleurs handicapés, et à la lutte contre le travail dissimulé. Dans la première affaire, les requérants contestaient l'obligation de prise en charge de l'hébergement collectif par le maître d'œuvre ou le donneur d'ordre lorsque des salariés sont soumis par un contractant ou un sous-traitant à des conditions d'hébergement incompatibles avec la dignité humaine. Dans la seconde, ils contestaient l'inégalité des règles applicables aux groupements d'employeurs d'une part et aux entreprises de travail temporaire d'autre part, quant aux règles de calcul du nombre de bénéficiaires de l'obligation d'emploi des travailleurs handicapés. Dans la troisième, c'est la règle selon laquelle le donneur d'ordre est solidairement responsable avec celui qui a fait l'objet d'un procès-verbal pour délit de travail dissimulé qui était mise en cause. Un peu à la manière du recours formé par la Société Saur SAS évoqué ci-dessus, ce qui est frappant dans ces affaires est la manière dont elles révèlent que, du côté des requérants, ce n'est pas tant pour garantir ou renforcer les droits et libertés constitutionnellement garantis que la QPC est utilisée, mais bien plutôt pour défaire des règles protectrices des travailleurs. Du côté du Conseil constitutionnel, en outre, chacune de ces trois affaires a, certes, mené à une décision de conformité (les dispositions législatives attaquées ont donc été confirmées comme conformes à la Constitution), mais sous réserve. Ainsi, c'est seulement sous la condition d'une interprétation sinon restrictive, du moins dûment cantonnée, que le Conseil a validé les dispositions du Code du travail relatives à l'obligation de fournir des conditions d'hébergement dignes ${ }^{45}$, la lutte contre le travail dissimulée ${ }^{46}$, l'obligation d'emploi des personnes handicapées ${ }^{47}$.

\section{Le rôle des filtres dans le façonnage de la catégorie " droits et libertés que la Constitution garantit »}

17 Une des choses que révèlent ces premiers éléments d'analyse, c'est donc l'émergence d'un espace nouveau à la faveur de la révision constitutionnelle de 2008: un droit constitutionnel des affaires ${ }^{48}$. On se rappelle combien les années 1990 avaient été dominées, en France, par le paradigme de la constitutionnalisation des branches du droit; mais par-delà la mutation intellectuelle qui causait alors la redéfinition de frontières et spécialités essentiellement académiques, c'est aujourd'hui un authentique espace social qui s'affirme, avec ses acteurs ${ }^{49}$, ses intérêts, ses procédures -parmi lesquelles la QPC occupe un des premiers rangs ${ }^{50}$. 
18 De cela, le Conseil constitutionnel lui-même ne saurait être tenu comptable ${ }^{51}$, au sens où, contrairement à ce qu'on observe dans d'autres pays, il ne choisit pas celles des QPC qui sont inscrites à son rôle. La QPC, on le sait, n'est en effet qu'improprement présentée comme une voie de recours permettant aux justiciables d'accéder directement au Conseil constitutionnel. Faisant écho à toutes sortes de réserves à cette hypothèse qui avait eu tout le temps de s'exprimer depuis les années 1980, la réforme constitutionnelle de 2008 a pris soin d'instituer des mécanismes de filtrage. Ainsi, si tout justiciable peut, au cours d'un contentieux, soulever l'inconstitutionnalité d'une disposition législative qui serait appliquée à son cas, il revient d'abord au juge $a$ quo de se prononcer sur le caractère sérieux du grief d'inconstitutionnalité soulevé. Ce n'est que s'il répond positivement à cette première interrogation qu'il saisira la juridiction suprême de l'ordre auquel il appartient (Conseil d'Etat ou Cour de cassation), lequel examinera de nouveau la question; deux filtres séparent donc le justiciable du Conseil constitutionnel... d'où l'importance de prêter attention auxdits filtres et au travail qu'ils opèrent en la matière pour déterminer ce qui constitue, ou non, matière à QPC c'est à dire, aux termes de l'article 61-1 de la Constitution, les cas dans lesquels il est valablement soutenu "qu'une disposition législative porte atteinte aux droits et libertés que la Constitution garantit ».

19 C'est donc plutôt vers les échelons réellement chargés du filtrage des QPC qu'il faut porter le regard pour chercher à comprendre les facteurs à l'œuvre dans cette orientation très économique d'une procédure promise à la protection des droits et libertés : juges a quo, puis Conseil d'Etat et Cour de cassation jouent en effet un rôle considérable dans la détermination des recours considérés à la fois comme " présentant un caractère sérieux » et comme mettant en cause un des "droits et libertés que la Constitution garantit ». Pour bien prendre la mesure du rôle que jouent les juges non constitutionnels dans l'émergence d'une nouvelle catégorie juridique (les «droits et libertés que la Constitution garantit »), il faudrait procéder à une analyse comparée des décisions de renvoi et des décisions de non-renvoi ${ }^{52}$ qu'ils prennent lorsqu'une QPC est posée devant eux. Un tel travail de dépouillement et d'analyse se situe clairement audelà de l'ambition du présent article. Mais il suffit, pour prendre la mesure des enjeux associés à cette étape-clef de la procédure de QPC, de rappeler à titre d'illustration la surprise qu'aura pu causer la décision de non-renvoi prise par la Cour de cassation après que la constitutionnalité des limites posées par le législateur à la liberté d'expression en matière de négationnisme lui eût été posée ${ }^{53}$. C'est cependant encore en-deçà de ces échelons juridictionnels du "filtrage » de la QPC qu'il faut s'intéresser pour comprendre cette physionomie particulière, très "droit des affaires ", de la QPC. Car en effet, si juges a quo et hautes juridictions jouent un rôle important dans le façonnage de la catégorie des QPC qui sont effectivement transmises au Conseil constitutionnel, ils travaillent à partir d'un matériau plus large, constitué par les QPC soulevées par les justiciables. Un travail de dissection mériterait donc d'être approfondi et mené de manière systématique dès cette étape, afin que puissent être mesurés les effets (l'effectivité) de la réforme constitutionnelle de 2008. Seul un tel travail pourrait permettre de mesurer réellement le degré de pénétration de la réforme instituant la QPC et la mesure dans laquelle elle est utilisée pour mettre les lois du pays à l'épreuve de standards constitutionnels de protection des droits de l'Homme. Dans cette attente, et sur la base des résultats du travail de dissection ici entrepris sur une année de contentieux QPC, force est de constater que cette nouvelle voie de recours est (i) largement captée par une catégorie bien particulière de justiciables (les personnes 
morales et, singulièrement, les entreprises) et (ii) largement mobilisée dans des contentieux très spécifiques (contestation, pour l'essentiel, de normes fiscales et/ou de réglementation de l'activité économique) et éloignés du champ des droits et libertés qui avait été mis en avant au moment de la révision constitutionnelle de 2008.

Dans le cadre du présent travail, qui s'attache à ouvrir des pistes de recherche plus qu'il ne prétend apporter des réponses, on n'a pu travailler que sur le second et ultime échelon du filtrage, celui opéré par le Conseil d'Etat et la Cour de cassation. On observe à cet égard que, sur les 66 décisions étudiées, elles font suite à un renvoi opéré par le Conseil d'Etat pour 38 d'entre elles (soit 57,5\% du total) et de la Cour de cassation pour 28 d'entre elles (soit $42,42 \%$ du total). Un affinement est possible sur la base des renvois opérés par la Cour de cassation, puisqu'on observe que nombre d'entre eux émanent de la chambre commerciale (10 sur 28) de la haute juridiction, ce qui semble annoncer une coloration très "droit des affaires » de la QPC. Mais à ces 10 renvois, il faudrait encore ajouter certains des 11 renvois émanant de la chambre criminelle, nombre d'eux soulevant en effet des questions dans le champ du droit pénal des affaires. On songe ainsi par exemple à celui ayant mené à la décision 2015-508 QPC, où était examinée la constitutionnalité des dispositions du Code de procédure pénale relatives au recours à la garde à vue prolongée de quatre-vingt-seize heures pour les infractions de blanchiment ou de recel du produit, des revenus, des choses provenant du délit d'escroquerie en bande organisée et pour les infractions d'association de malfaiteurs lorsqu'elles ont pour objet la préparation de ce même délit.

\section{Les moyens invoqués au soutien des QPC : quels sont « les droits et libertés que la Constitution garantit » dont la méconnaissance est contestée?}

Il est encore intéressant de s'intéresser aux moyens invoqués en matière de QPC. L'analyse serrée des 66 décisions rendues au cours de la période analysée ici révèle au moins trois enseignements. D'abord, la prépondérance incontestable du principe d'égalité dans le contentieux QPC : c'est, de loin, la méconnaissance de ce principe qui est invoquée par les requérants. Ensuite, l'investissement considérable qu'ils consentent dans les principes de la Déclaration des droits de l'Homme et du Citoyen -et singulièrement, dans ses articles 7, 8 et 9 qui sont couramment lus comme fondateurs du droit pénal moderne. Enfin, la part relativement congrue réservée à toutes les autres sources constitutionnelles des droits et libertés -ainsi que, parmi elles, la place éminente des libertés économiques.

Le principe d'égalité est invoqué, dans ses différentes déclinaisons (égalité devant la loi, égalité devant les charges publiques, égalité devant la justice...) dans pas moins de 36 des 66 décisions $(54,5 \%)$ étudiées. La prédominance du moyen est d'autant plus remarquable qu'il ne fait que rarement triompher l'argument au service duquel il est mobilisé. En effet, le Conseil ne retient une violation dudit principe que dans 7 des 36 cas. Dans tous les autres, il estime soit que la différence de traitement mise en cause par les requérants est justifiée par une différence objective de situation, soit qu'elle est justifiée par un objectif d'intérêt général. Ce moyen tiré de la méconnaissance du principe d'égalité semble toutefois apparaître comme particulièrement intéressant aux requérants qui soulèvent des $\mathrm{QPC}$; il est vrai qu'indépendamment de ses chances de 
succès, il est aisé à manier et permet la mise en cause toute situation dans laquelle un requérant peut arguer la soumission à une ou des règles différentes qu'un autre requérant avec lequel il peut raisonnablement estimer avoir des points communs ${ }^{54}$. Outre cette plasticité argumentative, le principe d'égalité peut également être considéré comme particulièrement prometteur en matière économique pour qui veut mettre en cause des réglementations au profit d'une plus grande liberté des acteurs. Un auteur avait d'ailleurs, en 2005, déjà, argué de ce que le principe constitutionnel d'égalité, articulé avec le principe de liberté, pouvait être lu comme fondant, en droit français, un principe général de libre concurrence ${ }^{55}$.

Ce sont ensuite les droits reconnus par la Déclaration des droits de l'Homme et du Citoyen qui figurent en bonne place dans l'année de contentieux QPC étudiée. Ainsi, le droit à un recours effectif ou le principe de séparation des pouvoirs (art. 16 DDHC), les principes de légalité, nécessité, et proportionnalité des peines ainsi que la présomption d'innocence (art. 7, 8, 9 DDHC) comptent parmi les règles dont la violation ou la méconnaissance est le plus souvent invoquée. En cela, le contentieux de la QPC confirme le mouvement, enclenché depuis le début des années 1970, d'acculturation profonde du droit français aux principes de la Déclaration -et à l'interprétation ou l'application de ce texte à des questions juridiques tout à fait contemporaines. On sait cependant la grande indétermination de nombre de ces principes qui rend complexe sinon réversible toute argumentation juridique qui prend appui sur eux ${ }^{56}$. On ne saurait être surpris dès lors que les voir aujourd'hui mis au service des causes les plus diverses ${ }^{57}$.

$\mathrm{Au}$ titre des normes plus clairement substantielles et plus clairement liées à la proclamation de droits et libertés fondamentales, le catalogue est épars. Ainsi, l'année étudiée aura vu invoquée la méconnaissance du droit au respect de la vie privée, de la liberté d'aller et venir, de diverses dispositions du préambule de la constitution de 1946 relative à des droits économiques et sociaux ou encore la liberté d'association, de manifestation et d'expression. Mais figurent également en bonne place les libertés économiques que sont le droit de propriété, la liberté contractuelle et la liberté d'entreprendre. Confirmant nombre d'éléments établis plus faut, cette dernière en particulier a connu un développement notable dans le contentieux constitutionnel, souligné récemment par différents auteurs qui insistent sur le fait qu'elle est finalement devenue une "liberté véritable, économiquement réaliste ${ }^{58}$ et que "le Conseil constitutionnel a triplé, depuis 2010, le nombre de censures fondées exclusivement sur la liberté d'entreprendre $\aleph^{59}$.

En outre, même si la présente étude entend s'intéresser aux usages de la QPC par les requérants plus qu'à ce que le Conseil constitutionnel en fait, il est intéressant de souligner que, sans qu'une mise en ordre statistique puisse prétendre être réellement probante sur un corpus restreint à une année de contentieux (seulement 66 décisions), on peut observer que non seulement la méconnaissance des libertés économiques est souvent invoquée mais encore que l'argument semble relativement efficace, puisqu'il est retenu par le Conseil constitutionnel dans un nombre non négligeable de cas ${ }^{60}$. Bien sûr, certains auteurs arguent de ce que la QPC n'aurait pas, ou très peu, fait progresser les libertés économiques ${ }^{61}$. Un tel constat va bien au-delà de l'ambition de la présente étude, qui voudrait simplement souligner le fait les entreprises et leurs conseils se sont allègrement emparés des libertés économiques. La liberté d'entreprendre sert ainsi, très concrètement, à plaider que l'obligation d'information des salariés qui pèse sur 
l'entreprise en cas de cession de parts y porte atteinte -et ce, de manière victorieuse puisque le Conseil constitutionnel juge effectivement que si le principe de l'obligation peut être justifié, sa sanction par la nullité de la cession est excessive ${ }^{62}$. Le droit de propriété sert à argumenter que le fait que le coût du recours à un expert ordonné par le CHSCT demeure à la charge de l'employeur même s'il exerce à l'encontre de cette décision son droit de recours aboutit à priver de garanties légale ledit droit constitutionnel $^{63}$. Quant à la liberté contractuelle, une atteinte disproportionnée y est portée atteinte par la procédure prévoyant les modalités de retrait d'un agrément d'un dépositaire de presse ${ }^{64}$. Ces différents éléments ne permettent certainement pas de remettre en cause la capacité de la procédure de QPC à servir également la protection des droits de l'Homme ; ils invitent toutefois à souligner le caractère non-automatique et non nécessaire des liens entre existence de la QPC et renforcement de la protection constitutionnelle de ces derniers. Car entre la règle et la réalité, il faut prêter attention à l'identité, aux intérêts et aux objectifs des acteurs sociaux qui s'en emparent.

Nombreux sont les auteurs soulignant à l'envi la « révolution » que consacre la QPC; et, pour la plupart, c'est une révolution qu'ils voient d'un bon œil. Dominique Rousseau exhorte ainsi : « de cette révolution-là, il ne faut pas avoir peur $»^{65}$. Soit. Mais s'il y avait dans la QPC un effet d'optique, un quiproquo? Si l'espace constitutionnel qu'elle fait naître n'était pas tant celui de l'individu que celui de l'entreprise, celui de la formation de droits et libertés à coloration largement économique, plus marqué par une logique des affaires que par une logique de la délibération : ne faudrait-il toujours pas en avoir peur? Le fait que la procédure de QPC apparaisse aujourd'hui largement mise au service d'intérêts économiques ne risque-t-elle pas de produire un effet bien différent de celui présenté initialement comme le but et la raison d'être de la QPC ? On souhaite ici ouvrir le débat et susciter des analyses plus approfondies sur ce qui peut être aujourd'hui décrit comme une "pente ${ }^{66}$ de la QPC.

\section{NOTES}

1. Même si, bien sûr, la route de la consécration d'une forme de contrôle a posteriori de la constitutionnalité de la loi a été longue; v. par ex. R. Badinter, «Aux origines de la question prioritaire de constitutionnalité", Revue française de droit constitutionnel, 2014, $\mathrm{n}^{\circ} 100$, pp. 775-782 ; et J.-H. Stahl, « La longue marche de l'exception d'inconstitutionnalité », in Mélanges en l'honneur du président Bruno Genevois, Dalloz, 2008, p. 993.

2. J.-L. Debré, cité par C. Maügué, «La QPC : 5 ans déjà, et toujours aucune prescription en vue », Les Nouveaux cahiers du Conseil constitutionnel, 2015, n 47, p. 11.

3. Les 5 ans de la QPC au Conseil constitutionnel: quelques chiffres, Avril 2015, http:// www.conseil-constitutionnel.fr/conseil-constitutionnel/francais/a-la-une/avril-2015-les-5-ansde-la-qpc-au-conseil-constitutionnel-quelques-chiffres.143503.html

4. Même si, en matière constitutionnelle, il faut concéder que la catégorie est largement impressionniste; sur ce point: V. Champeil Desplats, «Le Conseil constitutionnel a-t-il une conception des libertés publiques? », Jus Politicum, 2012, $\mathrm{n}^{\circ} 7$.

5. J. Bonnet, P.-Y. Gahdoun, La question prioritaire de constitutionnalité, PUF, 2014, Coll. Que sais-je? 
6. D. Rousseau, Droit du contentieux constitutionnel, 10è ed., Montchrestien, 2013, Coll. Domat Droit public, p. 44.

7. Pour une intéressante recherche sur les différents acteurs de la QPC et la mesure dans laquelle elle reconfigure le procès et la compréhension traditionnelle française du dualisme juridictionnel, voir E. Cartier, dir., La QPC, le procès et ses juges. L'impact sur le procès et l'architecture juridictionnelle, Dalloz, 2013, Coll. Méthodes du droit.

8. X. Dupré de Boulois, «La QPC comme supermarché des droits fondamentaux ou les dérives du contentieux objectif des droits", Revue des droits et libertés fondamentaux, 2014, chron. $\mathrm{n}^{\circ} 2:$ : Ce contentieux objectif des droits fondamentaux se particularise par la prohibition qui est faite au juge saisi du principal et à la juridiction de filtrage de soulever d'office un moyen d'inconstitutionnalité (art. 23-1 et 23-5 de l'ordonnance organique du 7 novembre 1958). L'idée est que «la question de constitutionnalité ne saurait permettre de purger les dispositions législatives de l'ensemble des inconstitutionnalités qu'elles seraient susceptibles de contenir, mais uniquement de celles qui font grief à une partie à l'instance » (...). Les requérants jouent donc un rôle essentiel dans la définition du périmètre de la question. Parce que le recours est dans "l'intérêt du droit", ils sont donc invités (incités) à soulever tous moyens tirés de la violation de droits et libertés constitutionnels de nature à révéler l'inconstitutionnalité de la disposition législative contestée ».

9. Contentieux fiscal dont il est établi qu'il représente une part quantitativement et qualitativement importante du contentieux QPC : v. G. Blanluet, «L'entreprise et la jurisprudence fiscale du Conseil constitutionnel », Les Nouveaux Cahiers $d u$ Conseil Constitutionnel, 2015, $\mathrm{n}^{\circ} 49$, p. 29 : «À s'en tenir à quelques données chiffrées, on constate que le contentieux fiscal n'est pas négligeable quantitativement et que, s'agissant du contrôle a posteriori en tout cas, les contribuables ont gain de cause plus souvent qu'on ne le pense. Au 31 décembre 2014, sur 380 décisions QPC rendues depuis le 1er mars 2010, 23 ont intéressé la fiscalité des entreprises, soit un peu plus de $5 \%$. Sur 145 décisions de censure, 16 ont été rendues en matière fiscale, dont 8 ont intéressé les entreprises. En ce qui concerne la fiscalité des entreprises, les dispositions déférées ont été jugées pleinement conformes à la Constitution dans moins d'un cas sur deux (47\%), contre 56,2\% toutes QPC confondues. Ainsi, dans la majorité des cas, le Conseil a prononcé une décision de non-conformité, totale ou partielle (34\%) ou a formulé une réserve d'interprétation (21\%)».

10. J. Bonnet, P.-Y. Gahdoun, La question prioritaire de constitutionnalité, op. cit..

11. Sur lequel on renvoie notamment à l'étude en trois parties de X. Dupré de Boulois, «Les droits fondamentaux des personnes morales", Revue des droits et libertés fondamentaux, 2011,

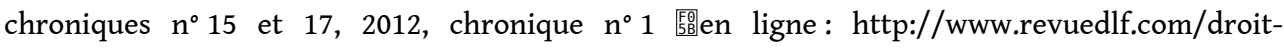
fondamentaux/les-droits-fondamentaux-des-personnes-morales---1ere-partie/ 閏.

12. L. Scheeck, La diplomatie des cours européennes. L'influence de la relation entre la Cour européenne des droits de l'Homme et la CJUE sur la construction européenne, Ed. de l'Université libre de Bruxelles, 2009 : « entre 1997 et 2006, la prépondérance à $52 \%$ de l'invocation de la Convention européenne en matière de politique de concurrence de l'Union Européenne est à première vue frappante. Sur un total de 224 décisions comprenant au moins une référence à la $\mathrm{CEDH}, 117$ sont relatives au droit de la concurrence. Les affaires concernant le droit institutionnel de l'Union Européenne viennent en deuxième position, avec seulement 18 arrêts ( $8 \%$ des affaires), suivies des 'affaires de fonctionnaires' (14 arrêts, $6 \%$ ), des affaires relatives à la libre circulation des personnes (10 affaires). $4 \%$ des affaires dans lesquelles apparaît la CEDH concernent la politique sociale, respectivement le rapprochement des législations ".

13. V. par ex. B. Morgan dir., The Intersection of Rights and Regulation: New Directions in Socio-Legal Scholarship, Ashgate, 2007.

14. V. sur ce point: V. Champeil-Desplats, D. Lochak, Libertés économiques et droits de l'Homme, Presses universitaires de Paris Ouest, 2011. 
15. Dont nombre d'acteurs répètent à l'envi que la QPC aurait contribué à en faire une authentique juridiction: V. R. Badinter, «Une longue marche du Conseil à la Cour constitutionnelle ", Cahiers du Conseil Constitutionnel, 2009, n² 25 ; ou encore J.-L. Debré, Ce que je ne pouvais pas dire, Robert Laffont, 2016, p. 74.

16. Au cours de la période considérée, les 66 décisions étudiées résultent de recours formés par 32 personnes morales et 34 personnes physiques. Parmi les 32 personnes morales, on dénombre : 1 recours formé par des communes, 24 recours formés par des entreprises ou fédérations d'entreprises, 2 recours formés par des syndicats, 4 recours formés par des ONG (défense des droits humains) ou associations (défense de l'environnement, ou Communauté rwandaise de France). Parmi les 34 personnes physiques, on dénombre 26 hommes, 4 femmes (dont Mme Kosciusko Morizet) et 5 requêtes conjointes (couples de requérants).

17. Sur cette possibilité, son régime (et notamment, l'absence de critères explicites) et sa pratique depuis 2010, v. A.-M. Lecis Cocco Ortu, «QPC et interventions des tiers: le débat contradictoire entre garantie des droits de la défense et utilité des amici curiae ", Revue française de droit constitutionnel, 2015 vol. 4, n 104), pp. 863-886.

18. Cette proportion est congruente avec les résultats de l'étude menée par A.-M. Lecis Cocco Ortu sur la période 2010-30 juin 2015. Elle recense en effet, sur les 413 décisions QPC rendues sur la période, 61 décisions dans lesquelles une "personne morale porteuse d'intérêts collectifs » a été admise en qualité de tiers intervenant. Or sur la base de la liste qu'elle fournit (note 39 de l'article cité), il apparaît que seule une douzaine d'intervenants sont des organisations de défense des droits et libertés, la cinquantaine d'autres étant composée de fédérations professionnelles (Union nationale des industries du taxi, par exemple), organismes nationaux divers (Conseil supérieur du notariat, ou Association des avocats pratiquant le droit de la concurrence, par exemple) ou associations nationales diverses (Union sociale de l'habitat par exemple).

19. 2015-471 QPC: Mme Kosciusko Morizet, élue du Conseil de Paris, a cherché, sans succès, à contester au nom d'un principe de publicité du vote les règles permettant aux assemblées locales de procéder à un vote à bulletins secrets.

20. L'une vise à mettre en cause les conditions de versement l'indemnité versée aux rapatriés anciens harkis, moghaznis et personnels des diverses formations supplétives ayant servi en Algérie (un recours similaire a également été formé par un requérant homme : 2015-504/505 QPC) et l'autre est relative à la suppression de l'arrérage de pension d'invalidité en cas d'activité professionnelle non salariée.

21. V. notamment F. Tulkens, « Droits de l'homme, droits des femmes. Les requérantes devant la Cour européenne des droits de l'homme ", in L. Caflisch et al. (éds.), Human Rights - Strasbourg Views. Droits de l'homme - Regards de Strasbourg, Kehl-Strasbourg-Arlington, N.P. Engel, 2007, pp. 423 et s.

22. Lire, comme en écho: "l'introduction de la QPC dans notre ordre juridique a éveillé un formidable élan; elle a fait du Conseil l'objet d'une soudaine popularité et les contribuables, à commencer par les entreprises, ont pu croire qu'auprès des juges de la rue Montpensier, ils trouveraient désormais une oreille attentive »: G. Blanluet, «L'entreprise et la jurisprudence fiscale du Conseil constitutionnel », op. cit. p. 29.

23. X. Dupré de Boulois, «La QPC comme supermarché des droits fondamentaux ou les dérives du contentieux objectif des droits », Revue des droits et libertés fondamentaux, 2014, chron. $\mathrm{n}^{\circ} 2$.

24. E. Piwnica, « L'appropriation de la QPC par ses acteurs », Pouvoirs, 2011, n 137, pp. 169-183.

25. O. Duhamel, « La QPC et les citoyens », Pouvoirs, 2011, n 137, pp. 183-191.

26. E. Piwnica, « L'appropriation de la QPC par ses acteurs », op. cit., p. 170.

27. O. Duhamel, « La QPC et les citoyens », op. cit., p. 185.

28. C. Maügué donne dans son étude les éléments statistiques suivants, construits cependant sur la base des décisions d'annulation rendues par le Conseil constitutionnel -ce qui ne permet pas de rendre compte des usages sociaux de la QPC, ie. des matières dans lesquelles les QPC sont 
soulevées : «Les dispositions annulées depuis le 1 mars 2010 concernent ainsi de manière très majoritaire le droit pénal ou la procédure pénale : sur 145 décisions censurant une disposition, 62 ont trait à cette matière. D'autres matières ressortent également, mais de façon quantitativement moins marquée: 13 décisions concernent le droit de l'environnement, 11 décisions le droit processuel (hors pénal), 11 décisions le droit fiscal, 5 décisions la santé publique. Les autres décisions de censure ont trait à des domaines variés : droit économique, droit des procédures collectives, droit des pensions, droit électoral, droit de la famille, droit de la fonction publique, etc. ». C. Maügué précise ainsi que «Cela ne signifie pas que la répartition par matière des QPC soulevées devant les juges ordinaires soit identique» et établit bien que «le principal pourvoyeur des QPC reste ainsi, devant le juge administratif, le contentieux fiscal, avec en 2014 $31 \%$ des QPC déposées en première instance et $24,5 \%$ des QPC en appel », in « La QPC, 5 ans déjà ", op. cit.. De la même manière, le bilan réalisé par le Conseil constitutionnel en avril 2015 (Les 5 ans de la QPC au Conseil constitutionnel: quelques chiffres, http://www.conseilconstitutionnel.fr/conseil-constitutionnel/francais/a-la-une/avril-2015-les-5-ans-de-la-qpc-auconseil-constitutionnel-quelques-chiffres.143503.html) se concentre sur les décisions de censure (dont il nous révèle qu'elle concernent en premier lieu le domaine du droit et de la procédure pénale, suivi par celui du droit et de la procédure fiscale). Or ceci ne permet pas de mesurer la part respective de ces domaines dans les QPC soulevées. En outre, il faut prendre conscience de ce que la catégorie « droit et procédure pénale » utilisée par le Conseil ne permet pas d'isoler ce qui, au sein de cette catégorie, relève du droit pénal des affaires.

29. À savoir : 10 décisions portant directement sur des dispositions du Code général des impôts (Il s'agit des décisions: 2016-538, 2015-520, 2015-515, 2015-503, 2015-481, 2015-479, 2015-475, 2015-474, 2015-473, 2015-466), 2 décisions portant sur des dispositions du Code des douanes (Décisions : 2016-537 et 2016-482), 2 décisions dirigées contre des dispositions des lois de finances rectificatives modifiant le CGI (Décisions : 2016-525 et 2015-475) et 2 dernières, relatives à des dispositions du Code de la sécurité sociale, mais relatives à des contributions obligatoires (l'une porte sur la CSG sur les contrats d'assurance vie multi-supports (Décision 2015-483) et l'autre sur la contribution des employeurs sur les rentes de retraite versées à leurs employés (Décision 2015-498)).

30. Cette catégorie compte 5 recours formés contre des dispositions du Code du commerce (Décisions : 2015-510, 2015-489, 2015-487, 2015-486, 2015-476), 1 recours relatif à des dispositions du Code monétaire et financier (Décision 2016-524), 3 recours relatifs au Code des transports (décisions 2015-516, 2015-484 et 2015-468/469/472), ainsi que des recours portant sur des dispositifs plus spécifiques (outre ceux cités dans le corps de l'article, v. aussi le recours contre la règlement des prix du pétrole dans les collectivités d'outre-mer (Décision 2015-507).

31. Décision 2015-511.

32. Décision 2015-480.

33. Décision 2015-524.

34. On insiste sur le fait qu'en cela, l'année ici étudiée ne paraît en rien spécifique ; ainsi, $X$. Dupré de Boulois avait déjà, en 2014, mis en lumière la manière dont la QPC pouvait être utilisée par des entreprises pour contester l'imposition de techniques de construction plus respectueuses de l'environnement (au nom de la liberté d'entreprendre: v. CC, 2013-317QPC, 24 mai 2013, Syndicat français de l'industrie cimentière), ou encore l'interdiction du travail du dimanche, in « La QPC comme supermarché des droits fondamentaux », op. cit..

35. http://www.saur.com/le-groupe/

36. Décision 2015-519: les critères de la représentativité des organisations professionnelles d'employeurs au niveau de la branche comptent notamment le critère selon lequel sont représentatives les organisations professionnelles d'employeurs dont les entreprises adhérentes à jour de leur cotisation représentent au moins $8 \%$ de l'ensemble des entreprises adhérant à des organisations professionnelles d'employeurs de la branche. V. aussi Décision 2015-2015-502. 
37. Comp. Les conclusions de l'étude de V. Bernaud, «Droit du travail et QPC : une rencontre fructueuse ?", in X. Magnon, X. Bioy, W. Mastor, S. Mouton, Le réflexe constitutionnel. Question sur la QPC, Bruylant, 2013, p. 37-62, qui porte quelques éléments au crédit du Conseil constitutionnel en matière de droit d'exercice collectif des travailleurs, mais souligne "que sa jurisprudence relative au droit individuel du travail reste assez pauvre », p. 45.

38. Décision 2015-496.

39. Décision 2015-523.

40. Décision 2015-533.

41. V. Bernaud, «La QPC a-t-elle changé le visage du droit constitutionnel du travail ? ", Droit Social, 2014, $\mathrm{n}^{\circ} 4$, pp. 317-324.

42. Décision 2015-517.

43. Décision 2015-479.

44. Décision 2015-497.

45. Décision 2015-517 : c'est seulement sous la réserve que l'obligation de prise en charge de l'hébergement pesant sur le maître d'œuvre ou le donneur d'ordre n'excède pas les salariés directement employés à l'exécution du contrat direct et la durée nécessaire à cette dernière qu'elle est validée.

46. Décision 2015-479 : c'est seulement sous la réserve que le donneur d'ordre puisse toujours contester la régularité de la procédure ayant mené, le cas échéant, à sa mise en cause solidaire avec celui ayant fait l'objet d'un procès-verbal d'infraction à la lutte contre le travail dissimulé que le dispositif est validé.

47. Décision 2015-497: si le Conseil estime que les entreprises de travail temporaire et les groupements d'entreprises sont placés dans des situations différentes et peuvent dès lors être soumis à des règles différentes, le Conseil précise que « les dispositions contestées ne sauraient, sans créer de rupture caractérisée de l'égalité devant les charges publiques, faire obstacle à ce que les salariés d'un groupement d'employeurs mis à disposition d'une entreprise utilisatrice soient pris en compte dans le nombre des bénéficiaires de l'obligation d'emploi des travailleurs handicapés, lorsqu'ils sont dénombrés dans l'assiette d'assujettissement du groupement à l'obligation d'emploi des travailleurs handicapés ".

48. A. Vauchez, «Les affaires devant le Conseil constitutionnel », http:// doyoulaw.blogs.liberation.fr ; et aussi, même auteur, L'espace privé de l'Etat, Presses de sciences po, à paraître 2016.

49. Et ce sont bien les avocats, les « détenteurs réels de l'initiative des QPC » : J. Bonnet, P.-Y. Gahdoun, La question prioritaire de constitutionnalité, op.cit.. V. aussi sur ce point B. Warusfel, «L'avocat comme acteur majeur du procès constitutionnel », in E. Cartier dir., La QPC, la procès, ses juges, op. cit., p. 86, qui cite une note du Conseil national des barreaux de 2009 : « Le succès de cette procédure repose sur la capacité de la profession d'avocat à s'en emparer et à la faire vivre. Chaque avocat, quel que soit son domaine d'activité, est concerné par la question de constitutionnalité ».

50. Ne serait-ce que du fait du caractère à la fois peu coûteux, rapide et extrêmement efficace, le cas échéant, de la procédure de QPC qui, en quelque mois, peut aboutir à l'abrogation pure et simple de dispositions législatives. V. là-dessus : E. Piwnica, «L'appropriation de la QPC par ses acteurs ", op. cit., p. 174 : « si les professionnels ont immédiatement adhéré à la QPC, c'est semblet-il parce qu'elle a répondu avec efficacité à un besoin réel selon une procédure empreinte de simplicité ».

51. Même si l'on peut relever un certain nombre d'indices de la volonté de l'institution de convaincre, précisément, le monde des affaires de son utilité ; v. par ex. l'interview consentie par le président J.-L. Debré, "Constitution, liberté d'entreprendre et concurrence ", Concurrences, $2014, n^{\circ} 2$. L'ancien président du Conseil constitutionnel relate par ailleurs dans son ouvrage récent que, s'il a répondu régulièrement à l'invitation d'Universités et autres associations locales 
de docteurs en droit pour prononcer nombre de conférences sur le Conseil constitutionnel et les libertés, il a aussi prêté une attention toute singulière aux entreprises, souhaitant développer des contacts avec elles. Il relate ainsi avoir invité Mme Laurence Parisot, présidente du MEDEF, à déjeuner en janvier 2008 ( «de tels contacts n'entrent pas dans les coutumes de cette maison, mais c'est précisément ce que je veux faire évoluer »), avoir organisé, en mai 2013, un « déjeuner avec une dizaine de chefs d'entreprise, que j'avais rencontré pour la préparation de notre décision sur la loi de finances 2012 ", ou encore un "petit déjeuner avec un chef d'entreprise important », qu'il « rencontre régulièrement » (J.-L. Debré poursuit : " pourquoi toujours fustiger les patrons, systématiquement les désigner tels que des ennemis, comme si nous étions revenus en plein XIXème siècle ») : J.-L. Debré, Ce que je ne pouvais pas dire, Robert Laffont, 2016, p. 29 p. 161 et p. 287.

52. D'autant que : «Les décisions rendues par les juridictions du filtre sont majoritairement des décisions de non-renvoi. Depuis l'entrée en vigueur de la question prioritaire de constitutionnalité le 1er mars 2010 jusqu'au 31 décembre 2014, le Conseil d'État et la Cour de cassation ont statué à 2313 reprises sur des QPC et leurs décisions se répartissent en 1856 décisions de non-renvoi (soit 80,3\%) et 456 décisions de transmission au Conseil constitutionnel (soit 19,7 \%) »: C. Maügué, « La QPC, 5 ans déjà », op. cit..

53. Pour rappel, la loi dite Gayssot $n^{\circ}$ 90-615 du 13 juil. 1990 réprimant le négationnisme (contestation de l'existence des crimes contre l'humanité tels que définis par le Trib. Nuremberg 1945) n'avait, en son temps, pas été déférée au Conseil constitutionnel par la voie de l'article 61 (contrôle a priori) -suscitant dès lors un intense débat sur sa constitutionnalité même. Il était donc attendu que le Conseil constitutionnel soit amené à se prononcer sur ce texte suite à l'instauration de la procédure de QPC. Toutefois, il a fallu s'y reprendre à plusieurs reprises pour que la Cour de cassation accepte de transmettre une QPC sur ce sujet; voir notamment la décision de non-renvoi : Cass. 7 mai 2010, $n^{\circ} 12008$ (puis, plus tard, une décision de renvoi Cass. 6 oct. 2015, n 4632 qui a donné lieu à la décision QPC 2015-512). On peut citer de nombreux autres exemples. Ainsi, alors même qu'elle décidait de transmettre une QPC en raison de l'imprécision réputée du délit de harcèlement sexuel tel qu'il figurait dans le Code pénal avant la loi 2012-954 du 6 août 2012 relative au harcèlement sexuel, avec les conséquences que l'on sait (v. CC, 2012-240 QPC, 4 mai 2012 et sur ce point le commentaire collectif REGINE, «Le harcèlement sexuel : une dépénalisation qui fait mauvais genre », http://regine-droit.blogspot.fr/2012/05/leharcelement-sexuel-une.html), la Cour de cassation refuse de transmettre une QPC relative au délit d'abus d'autorité, pourtant lui aussi rédigé de manière susceptible d'être qualifiée d'imprécise (v. art. 432-1 Code Pénal ; et v. Crim. 11 juill. 2012, n 11-88.664).

54. On se situe ici résolument du côté des requérants et de leurs avocats, sans méconnaître le fait qu'à l'inverse, le principe d'égalité est particulièrement difficile à manier pour le juge et ce, particulièrement dans des domaines comme le droit social, très largement construit sur des différenciations (v. sur ce point: P. Lokiec, «L'égalité devant la loi », Droit Social, 2014, p. 325 ; mais le constat pourrait être d'autant élargi que le droit social est loin d'être le seul domaine à présenter cette caractéristique: $\mathrm{O}$. Bui Xuan, Le droit public français entre universalisme et différentialisme, Economica, 2004).

55. D. Linotte, « Existe-t-il un principe général de la libre concurrence ?», Actualité juridique droit administratif, 2005, p. 1549.

56. V. le classique : D. Lochak, « Le Conseil constitutionnel protecteur des libertés?», Pouvoirs, $1986, \mathrm{n}^{\circ} 13$, p. 41.

57. Des auteurs plaident ainsi pour la reconnaissance d'un principe fondamental reconnu par les lois de la république comme libre concurrence -tout en reconnaissant qu'on peine à lui trouver un ancrage républicain: P. Hubert, A. Castan, «Droit constitutionnel et liberté de la concurrence », Les Nouveaux Cahiers du Conseil Constitutionnel, 2015, n 49, pp. 15-27. 
58. D. de Béchillon, «Le volontarisme politique contre la liberté d'entreprendre », Les Nouveaux Cahiers du Conseil Constitutionnel, 2015, $\mathrm{n}^{\circ} 49$, pp. 5-14, qui souligne la richesse d'appréhension de la liberté d'entreprendre qui se donne à voir dans des décisions telle que CC, 2012-285QPC, 30 novembre 2012 et la manière dont le Conseil en fait désormais une liberté susceptible de prévaloir même lorsqu'opposée, par exemple, à un impératif constitutionnel de protection sociale (v. notamment l'analyse proposée par l'auteur de la décision CC, 2013-672DC, 13 juin 2013).

59. A. Sée, «La QPC et les libertés économiques», La Semaine juridique. Edition générale, 2014, $\mathrm{n}^{\circ} 718$, étude 5.

60. Ainsi, invoquée dans 10 des décisions étudiées, la violation de la liberté d'entreprendre est retenue trois fois par le Conseil. Pour ce qui est du droit de propriété, sa méconnaissance a été invoquée 7 fois, et acceptée 3 fois. Quant à la liberté contractuelle, sa méconnaissance est invoquée par 3 requérants, et retenue 1 fois par le Conseil constitutionnel. On peut mettre ces éléments chiffrés en relation avec d'autres. Ainsi, des droits économiques et sociaux du préambule de 1946 ont été invoqués 6 fois, mais leur violation n'a été constatée par le Conseil qu'1 fois (et l'argument a donc été rejeté 5 fois). Pour ce qui est de la liberté d'expression, de manifestation ou d'expression dont la méconnaissance a été invoquée deux fois, ce fut toujours sans succès ; même chose pour la Charte de l'environnement. Quant au droit au respect de la vie privée, l'argument de sa méconnaissance a été retenu une fois et rejeté une autre fois.

61. V. aussi : P. Hubert, A. Castan, «Droit constitutionnel et liberté de la concurrence », op. cit., qui plaident pour une meilleure protection constitutionnelle du droit de la concurrence. De même, A. Sée souligne que le constat du triplement du nombre de censures du Conseil fondées sur la liberté d'entreprendre est, au mieux, cynique, dès lors que le point de départ était très faible - de sorte que le triplement ne signifie pas que la liberté d'entreprendre occupe effectivement une part considérable du contentieux constitutionnel. L'auteur Il déplore encore l'insuffisance des méthodes de contrôle déployées en la matière par le Conseil constitutionnel. Il nous semble néanmoins que cette déploration repose sur des analyses qui ne sont en rien spécifiques au contentieux de la liberté d'entreprendre et pourraient aisément être élargies à de nombreux autres domaines du contentieux des libertés. Quant aux éléments chiffrés, s'ils ne révèlent pas que la liberté d'entreprendre occupe ou représente une part considérable du contentieux constitutionnel, ils révèlent en revanche une évolution qui, elle, est bien à la hausse. V. A. Sée, «La QPC et les libertés économiques », op. cit.. Pour approfondir, on lira également M.L. Dussart, Constitution et économie, Dalloz, 2015, Coll. Nlle Bib des thèses, vol. 144.

62. Décision 2015-476.

63. Décision 2015-500.

64. Décision 2015-511.

65. D. Rousseau, Droit du contentieux constitutionnel, op. cit., p. 44.

66. X. Dupré de Boulois, « La QPC comme supermarché des droits fondamentaux », op. cit..

\section{RÉSUMÉS}

La QPC a six ans. Le bilan qu'on propose ici d'en faire adopte un angle particulier, qui consiste à interroger non pas tant la jurisprudence constitutionnelle en matière de QPC, que les usages qu'en font les requérants, tels qu'ils peuvent être appréhendés au moyen de l'étude des décisions rendues par le Conseil constitutionnel au fil d'une année de QPC. Qui soulève des QPC, dans quel 
but et en se fondant sur quels moyens : cherchant à apporter des éléments de réponse à ces questions, l'article attire l'attention sur l'importance des usages de la QPC par des entreprises qui se fondent sur leurs libertés économiques (liberté d'entreprendre, contractuelle...) pour poursuivre des objectifs parfois éloignés de la compréhension intuitive que l'on peut avoir des «droits et libertés que la Constitution garantit ».

The QPC -the new remedy for constitutional review that was introduced in the French constitution in 2008- is now six years old. As it seeks to assess its capacity to fulfill its constitutional mission (ensure the guarantee of constitutional rights), the article looks at one year of QPC litigation in order to understand what this particular procedure has come to mean for those who are its primary users : defendants. By drawing the profile of defendants, looking at the objectives they pursue throughout QPC actions and in what objectives, the article underlines the prevalence of corporate interest holders among defendants, who claim the respect of their economic freedoms (contractual freedom, freedom of enterprise...) in order to attain objectives that often appear only remotely connected to an intuitive understanding of the "rights and liberties that the Constitution guarantees ».

\section{INDEX}

Mots-clés : QPC, usages sociaux, droits fondamentaux, libertés économiques

Keywords : QPC, fundamental rights, economic freedoms

\section{AUTEUR}

\section{STÉPHANIE HENNETTE VAUCHEZ}

Stéphanie Hennette Vauchez est professeure de droit public à l'Université Paris Ouest Nanterre, où elle est directrice du M2 Droits de l'Homme et directrice-adjointe de l'UMR 7074 Centre de théorie et analyse du droit, équipe CREDOF. Ses travaux portent sur les droits de l'Homme, la bioéthique, la laïcité et les questions de genre. 